\title{
The Use of Conditional Lethal Cell Cycle Mutants for Temporal and Functional Sequence Mapping of Cell Cycle Events
}

JOHN R. PRINGLE

Division of Biological Sciences, The University of Michigan, Ann Arbor, Michigan 48109

\section{INTRODUCTION}

Conditional lethal mutants defective in particular steps of the cell division cycle ${ }^{1}$ have recently been isolated in several types of eukaryotic cells (Hartwell, '74; Nurse et al., '76; Frankel et al., '76; Orr and Rosenberger, '76; Morris, '76; Sato, '76; Basilico, '77; Siminovitch and Thompson, '78). Among the several uses to which such mutants can be put is the derivation of temporal and functional sequence maps of cell cycle events. By a temporal map, I mean very simply a diagram sum. marizi" g the temporal order of events during the cell cycle (fig. 1), such as could in principle be obtained by using a perfectly synchronous culture and methods for accurately and instantaneously monitoring each cell cycle event. Note that the temporal map per se provides no information on how the cell arranges for events to be carried out in an appropriate temporal order, or even on whether the temporal order indicated by the map is fixed or flexible. By a functional sequence map, I mean a diagram summarizing the functional interrelatedness of various cell cycle events. Figure 2 shows several possible examples. Note that a functional sequence map has implications for, but may not fully determine, the corresponding temporal map. For example, if three events occur in the dependent series A B $C$, then they must also occur in the fixed temporal order $A$ then $B$ then $C$. On the other hand, knowing that events $A$ and $B$ are in parallel pathways (fig. 2c) tells one nothing of their actual temporal order, and even sug. gests that the temporal order may be flexible.

An unambiguous discussion of the use of conditional lethal cell cycle mutants for constructing temporal and functional sequence maps requires the recognition that there are several different times in the cell cycle that are relevant to consideration of any particular mutant (fig. 3). First, there is the interval of the cycle during which the gene product in question normally carries out its essential function. Second, there is the interval of the cycle during which the gene product in question is normally synthesized. [Note that some gene products that function during discrete intervals of the cycle may be synthesized continuously throughout the cycle, while others (as shown in fig. 3) may be synthesized shortly before their times of function. Other patterns are also possible.] Third, there is the time in the cycle at which the first landmark event (an event detectable biochemically or morphologically with currently available techniques) that is known to be defective in the mutant normally occurs. This first known defective landmark event has been termed the diagnostic landmark by Hartwell ('74). Note that it may occur considerably later in the cycle than the time of function of the gene product in question. For example, one could have a mutant in which a failure of gene product function led to a failure to complete event $A$ (fig. 2a), but in which the failure of event $E$ to occur would be the first morphological or biochemical indication that cell cycle progress was arrested. Fourth, there is the terminal phenotype that each mutant cell ultimately attains after an extended incubation under restrictive conditions. This is shown as a branch

\footnotetext{
${ }^{1}$ Such mutants are appropriately termed cell cycle mutants. Conditional lethal mutants with more general metabolic defects (e.g. mutants defective in ATP generation or in protein synthesis) will of course also cease to progress through the cell cycle under restrictive conditions, although, in contrast to cell cycle mutants, they will dis play no distinctive terminal phenotype (see fig. 3 and associated discussion, below). Studies of such metabolic mutants may illuminate some aspects of the cell cycle (see ftn. 4), but it seems to me that a failure to distinguish clearly between such mutants and cell cycle mutants is an impediment to clear thinking about the cell cycle and the uses of mutants in analyzing it. I think this argument is not vitiated by the recognition that there are probably intermediate cases, that is, mutations in genes whose products function in two or three discrete cell cycle steps. Except as otherwise noted, all mutants discusged in this paper are cell cycle mutants sensu stricto.
} 


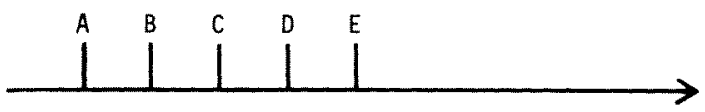

Time in the Cell cycle

Fig. 1 A temporal map of cell cycle events. The map indicates that event $A$ occurs earlier than event $B$, which occurs earlier than event $C$, and so on, but does not indicate the functional relations that may (or may not) exist among these several events. Note that "events" may be of several types. For example, event A might be "end of the period during which gene product $x$ functions"; event B might be "beginning of the period during which gene product $y$ functions"; event $\mathrm{C}$ might be "beginning of the period during which gene product $z$ is synthesized"; event $D$ might be "the completion of DNA synthesis"; etc.

from the cycle in figure 3 , in order to empha. size that the terminal phenotype cannot in general be regarded as a normal stage of the cycle at which cycle progress has suddenly become arrested (as by an instantaneous and perfect fixation procedure). For example, if the mutant gene product normally functions to catalyze event $\mathrm{C}$ (fig. $2 b$, c, or d), any event that normally occurs after event $\mathrm{C}$, but that is independent of $C$ (e.g., events D and $E$, fig. $2 b$, $c$, or d) will occur despite the nonoccurrence of C. This leaves the cell in a situation (i.e., $D$ and E completed, C not completed) that does not arise in the course of a normal cycle. Also, it is clear that most cell cycle mutants continue to increase their cell masses under restrictive conditions, so that a mutant cell blocked (for example) at nuclear division becomes much larger than a normal cell at the time of nuclear division.

Finally, for each conditional mutant allele of a particular gene there is an execution point (not shown in fig. 3). The execution point is defined operationally: it is that time in the cell cycle after which a shift from permissive to restrictive conditions can no longer prevent the mutant cell from successfully completing the current cell cycle. Thus, a cell that is prior to the execution point at the time of the shift to restrictive conditions arrests, with the appropriate terminal phenotype, without having divided under the restrictive conditions; a cell that is past the execution point at the time of the shift divides once successfully under the restrictive conditions, then arrests, with the appropriate terminal phenotype, in the subsequent cell cycle. It is crucial to realize that the execution point is an allele-specific, rather than a gene-specific, parameter. In other words, different conditional lethal alleles of the same gene can have vastly different ex- ecution points (Hartwell et al., '73). For example, for the gene described in figure 3 , a tight conditional-labile allele (in which the gene product lost its activity instantaneously and completely upon a shift from permissive to restrictive conditions) would have an execution point just at the end of the "time of gene product function". A leaky conditional-labile allele (in which the gene product lost its activity gradually or incompletely under restrictive conditions) would have an execution point earlier in the cycle; how early would depend on the degree of leakiness. A tight conditionalfor-synthesis ${ }^{2}$ allele (in which the mutant gene product retained its activity under restrictive conditions, but new active gene product could not be formed) would have an execution point at the end of the "time of gene product synthesis", or at whatever earlier time the cell had synthesized enough active gene product to see it through its current cell cycle. ${ }^{3}$

\section{USE OF CONDITIONAL LETHAL MUTANTS FOR TEMPORAL MAPPING}

At first glance, one might imagine at least three potentially fruitful approaches to the use of conditional lethal cell cycle mutants for the temporal mapping of cell cycle events.

\footnotetext{
${ }^{2}$ For the conditional mutants most commonly used (i.e., temperature-sensitive mutants), the corresponding terminology would be "temperature-labile" and "temperature-sensitive-for-synthesis",

${ }^{3}$ Some mutants display execution points in the cycle previous to the cycle of reference (or in even earlier cycles); that is, cells after the execution point divide successfully twice (or more) after a shift to restrictive conditions, before their daughters arrest, with the appropriate terminal phenotype, in the cycle of reference (Hartwell et al., '73). Hartwell (74) has described such mutants as "failing to exhibit first cycle arrest". Both intuition and the presence in most cases of other alleles of the same genes that do exhibit first cycle arrest (Hartwell et al., 73 ) suggest that such mutants are generally leaky alleles. However, it is certainly possible that some gene products are synthesized in adequate amounts, or even complete their functions, in anticipation of cycles that are to come.
}

Fig. 2 Examples of possible functional sequence maps for a set of cell cycle events. In each diagram, arrows connected head-to-tail denote that the associated events oc. cur in a dependent series. For example, diagram $a$ indicates that event $B$ is dependent upon event $A$; that is, event $B$ cannot be completed unless event $A$ has been completed. (However, event $A$ is not dependent upon event $B$, and can be completed even when completion of event $B$ is blocked.) Note that events in a dependent series obey the transitive rule: if (as in diagram $a$ ) $C$ is dependent upon $B$, which is dependent upon $A$, then $C$ is also dependent upon $A$. Events associated with the same arrow (e.g., events $F$ and $G$ in diagram $d$ ) are interdependent: neither can be completed while completion of the other is blocked Events not connected by a series of head-to-tail arrows (e.g., $\mathrm{A}$ and $\mathrm{B}$ in diagram $b ; \mathrm{A}$ and $\mathrm{B}$ in diagram $c$; or $\mathrm{C}$ and $\mathrm{D}$ in diagram $d$ ) are said to be independent, or in par. allel pathways : either event can be completed while completion of the other is blocked. 
a. Events occur in a dependent series:

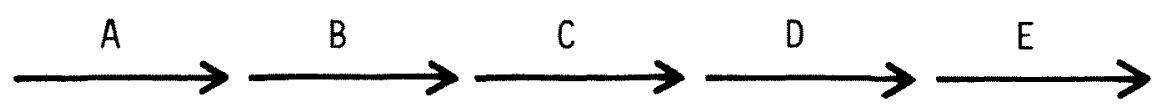

b. Events are independently triggered by some central "clock" (e.g., increasing cell mass):

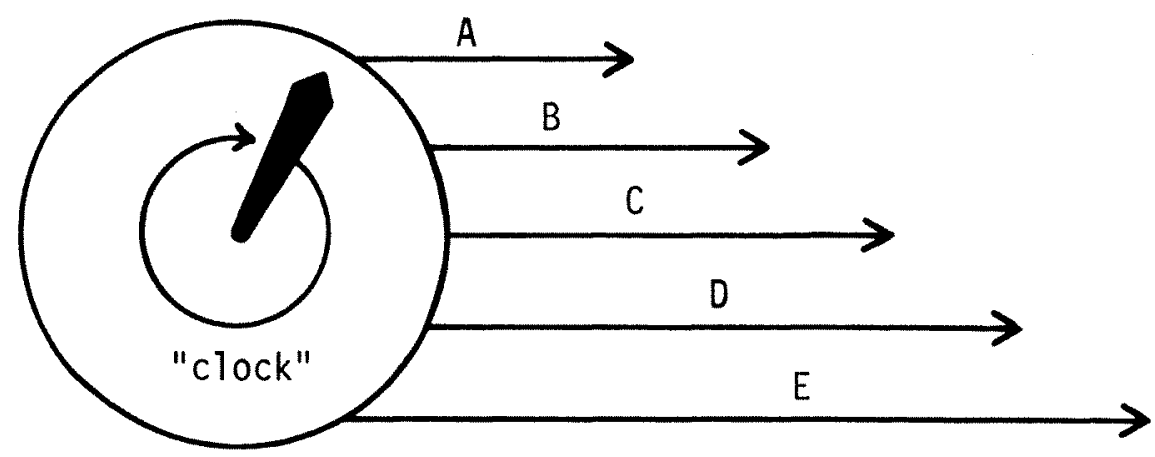

c. Events occur in two parallel pathways, each of which constitutes a dependent series:

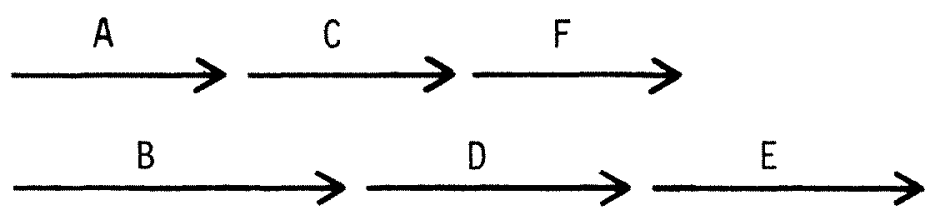

d. Events occur in two parallel pathways (D-E-H and C-F,G-I), each of which is dependent on an earlier event (B), and each of which is prerequisite for a later event (J):

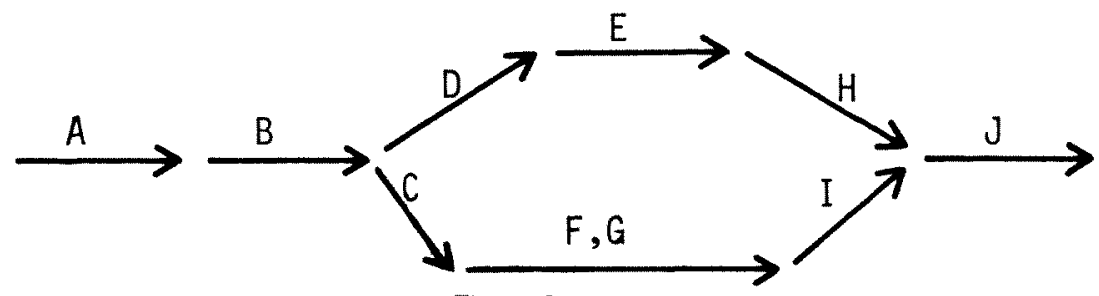

Figure 2 


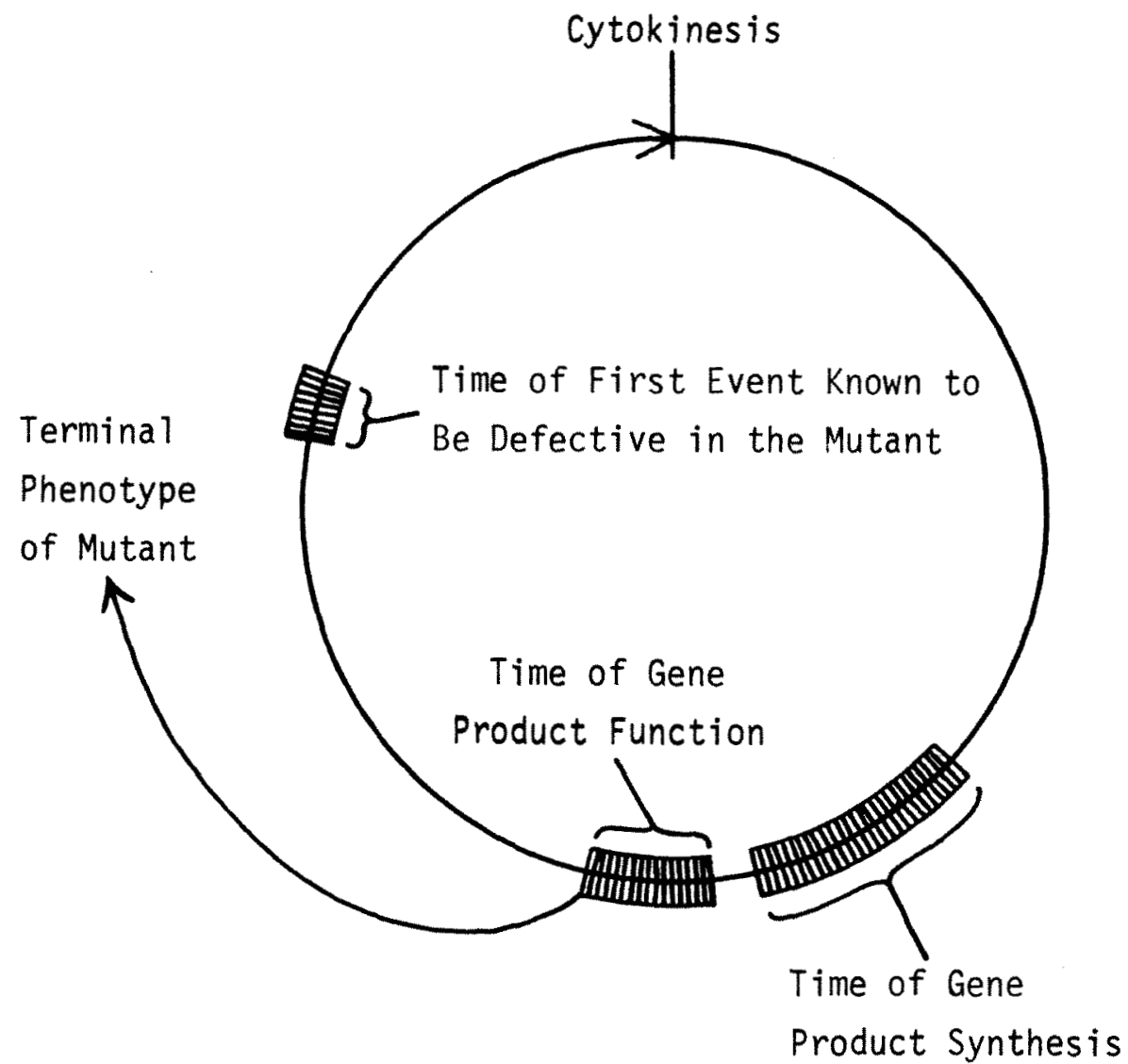

Fig. 3 Diagram of the cell cycle showing several of the times relevant to consideration of a particular hypothetical conditional lethal cell cycle mutant.

First, one might shift mutant cells to restrictive conditions and ask when a detectable interruption of cell cycle progress occurs. However, since the gene product identified by the mutation may be synthesized, and function, at any time prior to the first morphologically or biochemically detectable defect (see fig. 3 and the INTRODUCTION), such an experiment yields no temporal information beyond that obtainable (more reliably) with a wild-type strain. Second, one might allow mutant cells to arrest under restrictive conditions, then return them to permissive conditions and measure the time until some biochemically or morphologically detectable landmark event occurs. However, the hope of determining by such experiments when in the cycle (relative to the landmark event) different mutants are blocked seems to rest on the dubious and difficult to test assumption that all mutants recover immedi- ately (or, at least, that different mutants recover with identical kinetics) upon a return to permissive conditions. Even if a mutant gene product recovers its full activity immediately upon a return to permissive conditions, the time required to move from an abnormal terminal phenotype (see fig. 3 and the INTRODUCTION) to a landmark event may not bear any simple relation to the time required to move from the time of gene product function to the landmark event along the normal cell cycle path.

A third, and much more promising, approach to the use of cell cycle mutants for temporal mapping is the determination of mutant execution points. ${ }^{4}$ Execution points can be determined using synchronous cultures (for example, Culotti and Hartwell, '71). "Selection synchrony" is preferred to "induction 
synchrony" for the usual reasons (Mitchison, '71, pp. 25-57). [Note, in particular, that if parallel pathways of cell cycle events exist (fig. 2b-d), the treatment used for induction may synchronize the cells with respect to some pathways, but not with respect to the pathway in which the mutant gene product functions, and a grossly misleading measurement of the execution point would result.] Direct analysis of fractionated cultures (Mitchison, '71, p. 50; Wells and James, '72) will probably be a preferable alternative to synchronous cultures in execution point determinations, as in other applications.

Execution points can also be determined by following the development of individual cells after a shift to restrictive conditions, and relating whether or not each cell completes a normal cycle to its position in the cycle at the time of the shift to restrictive conditions (Culotti and Hartwell, '71; Hartwell, '74, '76). A limitation of this approach is that it requires a method (such as measurement of bud size in yeast) for assessing the cycle position of individual cells at the time of the shift to restrictive conditions. More ingenuity will be needed to overcome this limitation with organisms other than yeast, but the problems do not seem insurmountable. For example, time lapse cinematography of cells during the period prior to the shift to restrictive conditions should allow each cell whose development is followed after the shift to be characterized as regards the time of its last previous division under permissive conditions. It also seems possible that a combination of DNA. autoradiography plus cell size measurements might allow cells to be characterized at least as to whether they were in G1, S, or G2 at the time of the shift. It also seems likely that the continuing development of cell scanning devices and of techniques for monitoring biochemical markers of cell cycle progress will further enlarge the possibilities for applying the individual cell approach to organisms other than yeast.

The most widely applicable method of determining execution points is to measure the fraction of cells capable of dividing when an asynchronous population is shifted to restrictive conditions. From this fraction the execution point can be calculated by making the appropriate correction for the age distribution of the population (Hartwell et al., '73; Howell and Naliboff, '73; Howell et al., '75; Nurse et al., '76; Orr and Rosenberger, '76). This ap- proach has at least two inherent dangers. First, unless the results are corroborated by observations on single cells or on synchronous cultures, as described above, one must assume that the mutant of interest really has a well defined execution point (i.e., that the cells that divide after the shift to restrictive conditions really are the cells that were latest in the cycle at the time of the shift). [IIlustrations of such corroboration are provided by Hartwell et al. ('73); Howell and Naliboff ('73); and Hartwell ('74).J Second, one must assume that the mutant cells growing under permissive conditions have a normal cycle age distribution; given the nature of conditional lethal mutations, this assumption is certainly not always safe (Hartwell et al., '73; Pringle, '75; Nurse et al., '76).

Regardless of how execution point data are obtained, they must be interpreted with caution. It should be clear from figure 3 and the associated discussion (see INTRODUCTION) that, in principle, determining execution points can yield valuable information on the times in the cell cycle at which various essential gene products are synthesized, and on the times at which they complete their essential functions. However, it should also be clear that this information cannot be obtained un. less it is known which of the mutant alleles studied are tight conditional-labile, which are tight conditional-for-synthesis, and which are leaky (see INTRODUCTION). When the products of cell cycle genes have been identified, and quantitative assays for these products developed, such discriminations among alleles can be made by direct assays of the gene products of interest after shifts to restrictive conditions. Before this happy day, one must rely on indirect arguments, of which at least three seem likely to be useful. First, if the one or few available mutant alleles of a particular gene all fail to exhibit first cycle arrest, they should be presumed leaky until proven innocent (see ftn. 3), and strong conclusions should not be

\footnotetext{
${ }^{5}$ Note that inhibitors that affect specific cell cycle events also have execution points (defined analogously to those for cell cycle mutants). The following discussion of the determination and interpretation of execution point data applies to inhibitor execution points as well as to mutant execution points. Note also that mutants and inhibitors with more general metabolic defects (see ftn. 1 ) have "transition points" (Howell and Naliboff, '73 and Howell et al., "75) that are similar to execution points except for the lack of a distinctive terminal phenotype attained by all cells. Determination of these transition points can yield valuable information about the dependence of cell cycle events on general metabolism (e.g., at what point does the completion of cell division become independent of continued protein synthesis?). The following discussion of the determination and interpretation of execution point data applies also to these transition points.
} 
drawn from their execution points. Second, if multiple mutant alleles of a particular gene are available, one can compare their execution points. The latest of these execution points sets a limit on the time of gene product function: the gene product in question cannot complete its function earlier in the cycle than the latest known execution point. Moreover, if enough mutant alleles of a particular gene are a vailable that a clear pattern in the execution points is discernible (several execution points clustered at a certain time, all other execution points earlier in the cycle), one is probably justified in concluding that the clustered execution points mark the time at which the gene product completes its essential function. Finally, if mutants are isolated in a strain carrying a tight temperature-labile nonsense suppressor, then nonsense mutations in cell cycle genes will behave like tight temperature-sensitive-for-synthesis alleles, and determination of their execution points will allow statements about when the gene products are synthesized. [See Pringle ('75) for further discussion of the advantages of, and prospects for, this approach to mutant isolation.]

The arguments of the preceding paragraph are subject to one final caveat, namely that it is not clear to what extent genetic background differences may affect the execution points determined for a particular mutant allele. Since it seems likely that the effects may be significant, conclusions based on execution points should be tentative until the possible effects of genetic background differences have been assessed directly.

\section{USE OF CONDITIONAL LETHAL MUTANTS FOR FUNCTIONAL SEQUENCE MAPPING}

Functional sequence maps should be useful in at least two important ways. First, it seems clear that the events that comprise a cell division cycle must occur in a proper order if viable daughter cells are to be produced. The determination of functional sequence maps should illuminate the coordinating mechanisms that ensure that a proper order of cell cycle events is achieved (Hartwell, '74; Hartwell et al., '74). Second, functional sequence maps should provide clues that will be useful in elucidating the molecular nature of cell cycle events. For example, the determination of a dependent series A $B$ suggests that events $A$ and $B$ may be steps in a biochemical pathway (i.e., the product of $A$ would be the substrate for B), although other explanations are also possible. A less obvious, but equally pertinent, example is the conclusion of Hartwell (76) that two of the yeast "nuclear division" mutants must actually be defective in the fine details of DNA synthesis.

Three main approaches to the determination of functional sequence maps are available at the present time. Each approach has its limitations and its potential complications, as I have tried to indicate in the discussion that follows. This discussion is necessarily brief and somewhat abstract, and my list of potential complications is certainly not exhaustive. ${ }^{6}$ Thus, the reader interested in applying these approaches is urged both to spend some time imagining possible complications and to study carefully the several papers in which these approaches have been applied to real experimental systems (Coote and Mandelstam, '73; Jarvik and Botstein, '73; Hartwell et al., '74; Hereford and Hartwell, '74; Hartwell, '76; Nurse et al., '76; Frankel et al., '76).

For the discussion that follows, it is necessary to distinguish between the morphologically and/or biochemically defined landmark events (denoted $\mathrm{La}, \mathrm{Lb}, \mathrm{Lc}, \ldots$ ) and the primary defect events (denoted D1, D2,...) defined as the sites of the primary molecular defects due to particular cell cycle mutations or cell cycle-specific inhibitors. ${ }^{7}$ Thus, we will consider how to obtain information both on the functional interrelatedness of landmark events and on the functional interrelatedness of primary defect events. However, it should be clear that this distinction is of practical, rather than fundamental, significance; it simply reflects the fact that from the properties of mutants we can infer the existence of cell cycle events for which we have no direct morphological or biochemical assays at the present time. Thus, although a mutant must be analyzed in terms of the landmark events that do or do not occur, the primary defect event for the mutant may or may not be the same as its

\footnotetext{
${ }^{6}$ For example, I have not attempted to discuss in detail the complications that can arise when a gene product has two distinct functions (so that different mutant alleles of the gene might affect one function, or the other, or both). Note, however, that in this case (as in others - see below) the chances of drawing erroneous conclusions are reduced when multiple different mutant alleles of each gene have been analyzed.

7 Although written in terms of cell cycle mutants, most of the discussion that follows applies equally to the use of cell cycle-specific inhibitors, with the caveats that the danger of pleiotropic effects (see ftn. 5) is greater and that the benefits of being able to compare multiple different mutant alleles of the same gene are lacking.
} 
diagnostic landmark. (Figure 3 and the INTRODUCTION should help to make this clear.) In terms of functional sequence mapping, the diagnostic landmark must either be interdependent with the primary defect event (i.e., D1, La ) or subsequent to it in a dependent series (i.e., D1 … La ${ }^{8}{ }^{8}$ The same statement can be made about any other landmark that fails to occur in the mutant, but it is crucial to realize that the various landmarks dependent on $\mathrm{D} 1$ need not lie in the same dependent series (e.g., in fig. $2 \mathrm{~d}$ let $\mathrm{B}=\mathrm{D} 1, \mathrm{C}$ $=\mathrm{La}$, and $\mathrm{E}=\mathrm{Lb}$ ).

It is also important to note that the primary defect event for a particular mutant can be either in the synthesis or in the function of the mutant gene product, and that the synthesis of a gene product is a prerequisite for its function. Thus, if mutants 1 and 2 were conditional-for-synthesis and conditionallabile alleles, respectively, of the same gene, then a functional sequence map should show $\mathrm{D} 1, \cdots \mathrm{D} 2$ (or, in some cases, D1, D2 ). $\overrightarrow{\text { Various landmark events might be dependent }}$ on D2 (and hence, by the transitive rule, on D1 as well), or on D1 alone (e.g., in fig. $2 d$ let $B=$ $\mathrm{D} 1, \mathrm{E}=\mathrm{D} 2, \mathrm{C}=\mathrm{La}$, and $\mathrm{H}=\mathrm{Lb}$ ).

Comparing the phenotypes of single mutants. In this "single mutant method", one shifts strains carrying single cell cycle mutations to restrictive conditions and monitors the landmark events that do and do not occur (Hartwell et al.,'74; Nurse et al., '76; Frankel et al., '76). This method is the only one of those to be discussed that yields information about the functional interrelatedness of landmark events; sometimes it also yields information about the functional interrelatedness of primary defect events. Consider what can be learned from one such mutant, with a primary defect event D1. If two landmark events ( $\mathrm{La}$ and $\mathrm{Lb}$ ) both fail to occur under restrictive conditions, then each of them is dependent upon (or interdependent with) D1, but La and Lb may be interrelated in any of four possible ways ( $\mathrm{Lb}$ dependent on $\mathrm{La}, \mathrm{D} 1 \mathrm{La}$ $\mathrm{Lb}$; La dependent on $\mathrm{Lb}, \stackrel{\mathrm{D} 1}{\overrightarrow{\mathrm{Lb}}} \stackrel{\mathrm{La}}{\mathrm{L}}$; interdependence, $\mathrm{D} 1 . \stackrel{\mathrm{L} a, \mathrm{Lb}}{\mathrm{a}}$; independence, $\stackrel{\mathrm{D} 1}{\mathrm{La}} \mathrm{Lb})$. If $\mathrm{La}$ and $\mathrm{Lb}$ are clearly distinct in their temporal map positions, it should be possible to rule out interdependence and one of the two possible dependent relationships, but no discrimination between independence and the other dependent relation ship is possible. If a third landmark event $(\mathrm{Lc})$ occurs under the restrictive conditions, it may be related to $\mathrm{D} 1$ and $\mathrm{La}$ (or $\mathrm{Lb}$ ) in two possible ways:

$$
\stackrel{\mathrm{Lc}}{\longrightarrow} \stackrel{\mathrm{D} 1}{\longrightarrow} \stackrel{\mathrm{La}}{\longrightarrow} \text { or } \stackrel{\mathrm{Lc}}{\stackrel{\mathrm{Da}}{\longrightarrow}}
$$

In some cases, temporal map data may allow the former of these relationships to be ruled out. [For example, the $c d c 24$ mutants of yeast fail to form buds at restrictive temperatures, but do undergo nuclear division (Hartwell et al., '73, '74). Since nuclear division occurs much later in the cycle than budding, this leads immediately to the conclusion that budding and nuclear division are independent events.]

A more detailed analysis is possible only if two or more mutants with different spectra of blocked and permitted landmarks are available for comparison. ${ }^{9}$ If in mutant 1 (primary defect event D1) landmark event La occurs but landmark event $L b$ does not, and in mutant 2 (primary defect event D2) Lb occurs but La does not, then a strong conclusion is possible: $\mathrm{La}$ and $\mathrm{Lb}$ are independent, as are $\mathrm{D} 1$ and D2. [That is, in the functional sequence map, $\left.\frac{\mathrm{D} 1}{\mathrm{D} 2} \cdots \frac{\mathrm{Lb}}{\mathrm{La}}.\right]$ The only apparent complication is the possibility that in one mutant a normal control mechanism has broken down. For example, in budding yeast, several mutations block cytokinesis but allow nuclear division, while every mutation or inhibitor tested to date that blocks nuclear division also blocks cytokinesis (Hartwell et al., '74). This has suggested (see below) that cytokinesis is dependent on the prior completion of nuclear divi-

\footnotetext{
"Throughout the following discussion, it is assumed that the category of landmark events and the category of primary defect events are mutually exclusive. Thus, each landmark event can be monitored biochemically or morphologically, but can only be blocked indirectly, by blocking a primary defect event upon which it is dependent; in contrast, each primary defect event can be directly and specifically blocked, but can only be monitored indirectly, by observing its effects on landmark events. This assumption is convenient for discussion, and is appropriate for functional sequence map. ping efforts at the present time, since the molecular nature of the primary defect event is known for yery few cell cycle mutants. How. ever, it should be clear that the availability of events that are simultaneousiy landmark events and primary defect events will greatly facilitate the determination of functional sequence maps. In particular, analysis of the functional interrelatedness of landmark events by the "single mutant method" (next section) will be simpler, and will provide direct evidence for the existence of dependent series of landmark events.

9 If the two mutants have the same spectra of blocked and permitted landmarks, but have terminal phenotypes that differ in some other way, a limited conclusion is possible, namely that their primary defect events are not interdependent. Note, however, that an apparent identity of terminal phenotypes does not necessarily imply that the primary defect events are interdependent, since the apparent identity might mean only that our asagy procedures are in sufficient to detect the differences in molecular events completed under restrictive conditions.
} 
sion. The discovery at this point of a new mutant in which cytokinesis occurred despite the nonoccurrence of nuclear division would be less likely to suggest that cytokinesis and nuclear division are independent (the conclusion to which the above analysis would lead us) than that in the new mutant the normal control mechanism linking cytokinesis to nuclear division is broken down (Nurse et al., '76). As should be apparent from this example, the best defense against this complication is to analyze as many different mutants with effects on La or $\mathrm{Lb}$ as possible. The occasional mutants in which normal controls are broken down should be revealed as exceptions to the general patterns of apparent dependence relationships.

Another common situation is that in $\mathrm{mu}$ tant 1 neither La nor Lb occurs, while in $\mathrm{mu}$ tant 2 La occurs but Lb is blocked. Such data have generally been interpreted to mean that Lb is dependent on La (Hartwell et al., '74; Nurse et al., '76); the presumed dependence of cytokinesis upon nuclear division (see preceding paragraph) is a case in point. However, such an inference is not fully justified. Indeed, any of the following functional sequence maps is compatible with these data:

$$
\begin{aligned}
& \stackrel{\mathrm{D} 1}{\mathrm{La}} \stackrel{\mathrm{D} 2}{\longrightarrow} \stackrel{\mathrm{Lb}}{\longrightarrow}
\end{aligned}
$$

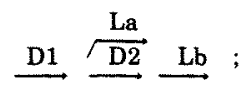

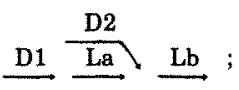

$$
\begin{aligned}
& \stackrel{\mathrm{D} 2}{\mathrm{D} 1} \stackrel{\mathrm{Lb}}{\longrightarrow} \stackrel{\mathrm{La}}{\longrightarrow}
\end{aligned}
$$

If $\mathrm{Lb}$ clearly precedes $\mathrm{La}$ on the temporal map, possibilities (1) and (3) can be eliminated. Otherwise, further information on the functional interrelatedness of $\mathrm{La}$ and $\mathrm{Lb}$ can only come from characterizing additional mutants. The discovery of a mutant in which Lb occurs but $\mathrm{La}$ is blocked would suggest that possibilities (1) and (3) could be eliminated, although the possibility that a normal control had broken down (see preceding paragraph) would need to be considered. If no such mutant were found, while many different mutants in which neither La nor Lb occurred were found, the tentative inference that either map (1) or map (3) was correct would be justified. [This is in fact the situation for sev. eral of the dependence relationships deduced by Hartwell et al. ('74) and Nurse et al. ('76), including the dependence of cytokinesis on nuclear division as mentioned above.] Note that neither temporal data nor the characterization of additional mutants can discriminate between maps (1) and (3) or between maps (2) and (4). Thus, to determine whether D1 and D2 are independent or occur in a dependent series one must use other methods, of which the two most useful are described below.

The method of comparing the phenotypes of single mutants has several pronounced merits. It provides information about the functional interrelatedness of landmark events, and its resolving power will increase as the number of events in the landmark category (i.e., events for which biochemical or morphological assays are available) increases. The method utilizes strains carrying single mutations, and thus it is relatively easy to apply in systems in which genetic manipulation is difficult. [Note, however, that some capacity for genetic analysis is desirable, since it is important to provide direct evidence that the effects studied in a particular strain really are due to a single mutation.] There is no requirement that the mutants used remain viable during incubation under restrictive conditions, and leaky mutants should lead to the same conclusions as tight ones. Conditional-for-synthesis alleles should lead to the same conclusions about landmark events as do conditional-labile alleles, unless some landmarks are dependent on the synthesis, but not on the function, of the gene product. Even in this case, the conclusions drawn from study of a conditional-for-synthesis allele should be compatible with those drawn from study of a conditional-labile allele. Moreover, the method seems immune to complications due to labile products of gene product function (cf. the discussion of the "reciprocal shift method," below).

However, it should be clear that this method also has its limitations and potential complications. It is crucial to remember the limitations imposed by the fact that the diag. nostic landmark for a given mutant cannot be assumed to be its primary defect event; in particular, note that the conclusion that one landmark event is dependent on another must always be tentative because of the chance that the mutant that would demonstrate 
independence has not yet been isolated [recall maps (1)-(4), above]. ${ }^{10}$ On the other hand, the conclusion that two events are independent should be tentative because of the possibility that a mutant has been isolated in which a normal control mechanism has broken down. Additional complications could arise if some sets of cell cycle events are triggered independently by a "clock" (fig. $2 \mathrm{~b}$ ); in this case, a mutant that caused the "clock" to stop at a particular point could clearly give a misleading impression that later landmark events were dependent upon earlier ones. With regard to each of these potential complications, it is clear that the probability of drawing valid and interesting conclusions about the functional interrelatedness of landmark events increases directly as the number of mutants analyzed.

Finally, it should be clear that this method can provide only limited information about the functional interrelatedness of primary defect events. In some cases it can be concluded that the primary defect events are independent, in other cases (see ftn. 7) that they are not interdependent, and in other cases no conclusion can be drawn. It is crucial to realize that the conclusions that can be drawn refer to the primary defect events per se. In many cases, the primary defect event can be simply equated to gene product function, in other cases it can be equated to gene product synthesis, but in still other cases the relationship may be more complicated. [For example, note that conditional-for-synthesis alleles that were deficient, respectively, in polypeptide chain formation, an early stage of polypeptide chain folding, or a late stage of polypeptide chain folding, should show different dependence relationships with events that were dependent, respectively, on mere presence of polypeptide chain, on presence of partly folded polypeptide chain, or on presence of fully folded polypeptide chain.l The probability of correctly translating a functional sequence map of primary defect events into a functional sequence map of gene product synthesis events and gene product function events clearly increases with the number of mutants (including the number of different alleles of each gene) analyzed.

Comparing the terminal phenotypes of two single mutants to that of the corresponding double mutant. This "double mutant method" provides useful information about the functional interrelatedness of primary defect events in cases in which two mutants have the same spectra of blocked and permitted landmarks events, but have terminal phenotypes that differ in some other way (Coote and Mandelstam, '73; Hereford and Hartwell, '74). ${ }^{11}$ To apply the method, it is necessary to have two single mutant strains carrying different cell cycle mutations, as well as the double mutant strain carrying both mutations. It must be possible to impose restrictive conditions for both mutations simultaneously; thus, two temperature-sensitive mutations make a satisfactory pair, while a temperature-sensitive mutation and a cold-sensitive mutation do not. Each of the three strains is simply shifted to restrictive conditions, and their terminal phenotypes are compared.

Interpretation of the results depends on the assumption that the terminal phenotypes of the single mutants differ because these strains complete different sets of normal cell cycle events under restrictive conditions, rather than because one of the mutant alleles results in a gene product that has acquired an abnormal activity. [This assumption is difficult to test with any particular mutation. However, if several different mutant alleles of the same gene all behave similarly, the assumption is probably valid (Hereford and Hartwell, '74).] Granted this assumption, the logic of interpretation is as follows. First, the fact that the two single mutants have different terminal phenotypes immediately eliminates the possibility that their primary defect events are interdependent (see also ftn. 8). Second, if the two primary defect events occur in a dependent sequence D1 D2, then the terminal phenotype of the double mutant should be identical to that of mutant 1 , since mutant 2 completes D1 (but not D2) under restrictive conditions, while both mutant 1 and the double mutant complete neither D1 nor D2. Third, if the two primary defect events are independent, then the terminal phenotype of the double mutant (which completes neither

\footnotetext{
${ }^{10}$ Recall footnote 7.

11 The method is also sometimes useful in cases in which the landmark events blocked in one mutant are a subset of those blocked in the other. For example, in the case described by maps (1)-(4) fpreceding section, a difference between the terminal phenotype of the double mutant and that of mutant 1 would be evidence that either map (3) or map (4) was correct (i.e., that D1 and D2 were indepen. dent), by the same reasoning (and subject to the same qualifications) as described in the text. Note, in particular, that a failure to find such a difference would not be good evidence in favor of map (1) or (2).
} 
D1 nor D2 under restrictive conditions) should be different both from that of mutant 1 (which completes D2 but not D1) and from that of mutant 2 (which completes D1 but not D2). However, it is possible that the resolving power of presently available techniques may not be sufficient to distinguish the unique terminal phenotype of the double mutant from that of one or the other of the single mutants. Thus, the observation that the double mutant has a unique terminal phenotype constitutes strong evidence that the primary defect events are independent. In contrast, the observation that the terminal phenotype of the double mutant is indistinguishable from that of mutant 1 rules out the dependent sequence D2 D1, but cannot distinguish between the dependent series D1 D2 and the possibility that D1 and $\mathrm{D} 2$ are independent.

The preceding comments on the expected behavior of the double mutant strains were based on the tacit assumption that at the time of the shift to restrictive conditions most cells in the population were either prior to both relevant execution points or (what amounts to the same thing) after both relevant execution points. If this assumption is invalid, misleading results can be obtained. In particular, if the primary defect events were related in a dependent series $\mathrm{D} 1, \mathrm{D} 2$, and the execution point for mutation 1 preceded that for mutation 2 by a wide margin, then an asynchronous population of the double mutant would contain many cells (conceivably even the bulk of the population) that are between the two execution points. These cells will complete D1 but not $\mathrm{D} 2$ under restrictive conditions, and their terminal phenotype will be the same as that of the single mutant 2, seemingly implying that the sequence $\mathrm{D} 1, \mathrm{D} 2$ can be ruled out. This danger can be avoided by experimentally measuring the execution points of the two single mutants. If these are too far apart, it may be possible to use other alleles of the same genes that have more favorable execution points. Alternatively, it should be possible to use synchronous cultures of the double mutant to ensure that most of the individual cells really do fail to complete both primary defect events under the restrictive conditions. It is important to note that, so long as the execution points are not too far apart, the method gives the same answer regardless of the actual order of the execution points. Thus, if conditional-labile alleles are used, the results should suggest the correct order of gene product function, even if one (or both) of the mutant alleles used is somewhat leaky. Of course, if the mutant alleles used are conditional-for-synthesis, the conclusions drawn may be appropriate for the actual primary defect events rather than for gene product function events.

In summary, when it can be applied, the double mutant method is a valuable approach to determining the functional interrelatedness of primary defect events. It can be applied using pairs of mutants of the same type (e.g., pairs of the relatively plentiful temperature-sensitive mutants), although other combinations are also possible (e.g., one mutant plus an inhibitor). There is no requirement that the mutants retain viability during incubation under restrictive conditions, and, provided reasonable precautions are taken to ensure that execution points are not too far apart, leaky mutants lead to the same conclusions as do tight ones. The method does require a significant genetic capability, since it is necessary to obtain (and to prove that you have obtained) both of the single mutant strains and the double mutant strain. This requirement may be a serious obstacle to attempts to apply this method to some experimental systems, such as animal cells. Moreover, the method is inherently limited in that it can provide strong evidence for the independence of two primary defect events, but not for the existence of a dependent sequence.

Reciprocal shift experiments. The most generally useful approach to the determination of functional sequence maps of primary defect events is the "reciprocal shift method" (Jarvik and Botstein, '73; Hereford and Hartwell, '74; Hartwell, '76). The method can be used when two different cell cycle-specific blocks can be independently imposed on the same strain. [For example, a temperature-sensitive cell cycle mutant can be used in conjunction with a cell cycle-specific inhibitor (Hereford and Hartwell, '74; Hartwell, '76), or a strain carrying both a temperature-sensitive cell cycle mutation and a cold-sensitive cell cycle mutation can be used (Jarvik and Botstein, '73). In the latter case, it is essential that some intermediate temperature be permissive with respect to both mutations.] It is also necessary that each block be reversible; that is, the cells must remain viable (as judged by their ability to resume cell cycle progress upon a return to permissive conditions) during a period of incubation under restrictive condi- 
tions. (It is not strictly necessary that recovery after a return to permissive conditions be rapid, although this will generally give cleaner experimental results.) It is, of course, easy to ascertain experimentally whether this condition is met.

Given a suitable pair of mutants (and/or inhibitors), two separate experiments must be performed. In the first experiment, the cells are initially exposed to conditions that are restrictive for mutation or inhibitor 1 (so that primary defect event D1 cannot be completed), but permissive for mutation or inhibitor 2 (so that primary defect event D2 can be completed unless it is dependent on D1). The cells must be left under these conditions long enough that all cells in a normal population would have completed event D2. Then, in order to determine whether $\mathrm{D} 2$ has in fact been completed by the cells in which $\mathrm{D} 1$ has been blocked, the cells are shifted to conditions permissive for D1 but restrictive for D2, and their ability to carry out cell division (or any other landmark event that is dependent both on D1 and on D2) is monitored. If the cells are able to carry out the landmark event under conditions restrictive for D2, this event must have been completed during the first incubation, and the functional sequence map should show $\underline{\mathrm{D} 2} \longrightarrow \mathrm{D} 1$ or $\frac{\mathrm{D} 1}{\mathrm{D} 2}$. If the cells are unable to carry out the landmark event during the second incubation, then D2 must not have been completed during the first incubation, and the functional sequence map should show D1 D2 or D1, D2. In either case, it is possible to distinguish between the two remaining possibilities by performing a second experiment, in which the first incubation is restrictive for D2 but not D1, and the second incubation is restrictive for D1 but not D2.

It is worth noting that the logic of the reciprocal shift method is really quite similar to that of the single mutant method. However, rather than simply determining what landmark events occurred during the first incubation, one must use a second incubation to determine what primary defect events occurred during the first incubation. Since one is asking about relations between primary defect events, which can be directly and specifically blocked, it is possible to obtain direct evidence for the existence of dependent series (cf. discussion of the single mutant method, above). The reciprocal shift method provides no new information in cases in which the single mutant method demonstrates independence of landmarks and primary defect events. In cases in which the single mutant method provides little or no information (two mutations block exactly the same landmark events, or the landmark events blocked by one mutation are a subset of those blocked by the other), the reciprocal shift method can provide additional information about the functional interrelatedness of primary defect events but not of landmark events. For example, in the case described by maps (1)-(4) (see section on the single mutant method), the reciprocal shift method could rule out either maps (3) and (4) or maps (1) and (2), but could not distinguish between map (1) and map (2), or between map (3) and map (4).

In interpreting the results of reciprocal shift experiments, several potentially serious complications must be kept in mind. First, it should be clear that the conclusions obtained relate to the primary defect events per se; as mentioned above (last paragraph of the section on the single mutant method), these can usually, but not always, be simply equated either to gene product synthesis events or to gene product function events. (It is a useful exercise to try to define the circumstances that would allow the functional sequence gene product synthesis gene product function to be deduced from reciprocal shift experiments involving a conditional-labile allele and a conditional-for-synthesis allele of the same gene.) Moreover, the situation is further complicated by the fact that what the reciprocal shift experiments really provide is an "order of executability": they tell us whether the execution point for block 2 can be passed while block 1 is imposed on the cells, and vice versa. This order of executability may or may not be the same as the functional sequence of the primary defect events. For example, suppose that the primary defect events for a pair of mutants actually occur in the dependent sequence D1 D2, but that mutant 1 is tight, while mutant 2 is sufficiently leaky that its execution point is significantly earlier in the cell cycle than the time at which event $D 1$ occurs. Under these conditions, reciprocal shift experiments would show that execution point 2 could be passed while event D1 was blocked, and vice versa, suggesting (erroneously) that D1 and D2 were independent. In a second important example, suppose that D1 and D2 are actually interdependent, but that mutant 1 is tight while mutant 2 is sufficiently leaky 
that its execution point can be passed while D1 is blocked. Now the results of reciprocal shift experiments would suggest (erroneously) the functional sequence $\mathrm{D} 2, \mathrm{D} 1$. Since the order of executability is of considerably less intrinsic interest than the functional sequence map of primary defect events, it is clearly desirable to do reciprocal shift experiments with tight conditional-labile or conditional-for-synthesis alleles. The problem of identifying these tight alleles has been discussed above (see section on temporal mapping), but it is worth reiterating that the odds improve directly as the number of mutants available for analysis.

Another possible complication is that one gene product may produce a substance that is labile, or induce a state that is transitory (Jarvik and Botstein, '73). In the course of a normal cell cycle, this substance or state would be quickly operated upon by subsequent gene products. However, the protocol for reciprocal shift experiments demands that the cells be held in the first incubation long enough for all cells to complete the permitted event (if they can). Thus, it is possible that during the first incubation the cells will in fact have completed the permitted event, but that the substance or state produced by completing the permitted event will have been dissipated in most cells by the time that the shift to the sec. ond incubation is made. The result would be a failure to complete the later landmarks during the second incubation, and the erroneous conclusion that the permitted event had not been completed during the first incubation because it was dependent on the restricted event. For example, if the actual functional sequence of primary defect events were $\mathrm{D} 1$ D2, but D1 produced a labile product, the erroneous conclusion that D1 and D2 were interdependent could be drawn (Hereford and Hartwell, '74). Similarly, if D1 and D2 were independent, but D1 produced a labile product, the erroneous conclusion that D1 was dependent on $\mathrm{D} 2$ ( $\mathrm{D} 2 \mathrm{D} 1$ ) could be drawn. The possible complication under discussion is a particularly nasty one, since comparing the results obtained with different mutant alleles of the same genes is not likely to reveal the existence of the problem. The only apparent protection against this complication is to minimize the opportunity for lability to be expressed, by minimizing the length of the first incubation (presynchronized cultures would be useful in this regard), and by using (where possible) mutants that recover quickly upon a return to permissive conditions.

Application of the reciprocal shift method will probably be limited primarily by the availability of suitable pairs of mutant and inhibitor blocks that can be independently and reversibly imposed. The approach with the widest potential applicability seems to be the use of double mutants containing both a temperature-sensitive and a cold-sensitive mutation, as pioneered by Jarvik and Botstein ('73) in studies of bacteriophage. Jarvik and Botstein ('75) subsequently described what should be a powerful approach to collecting large numbers of temperature-sensitive and cold-sensitive mutants suitable for use in reciprocal shift experiments. However, application of these approaches demands a significant capability for genetic analysis. This should not prevent their application to bacteria, yeast, and other microorganisms, but will probably be a serious obstacle to their use with animal cells. It may be feasible to start with a parent cell line that is temperaturesensitive but not cold-sensitive, select coldsensitive cell cycle mutants in this genetic background, and then do reciprocal shift experiments on the putative double mutants. However, the problems of showing that one really has mutations in two different genes, and of relating the results obtained with one strain to those obtained with others, do not seem trivial.

In contrast, an approach that should be readily applicable to animal cells as well as to other systems is the use of inhibitors in conjunction with temperature-sensitive or coldsensitive mutations. The applicability of this method should be limited only by the number of good cell cycle-specific inhibitors that can be obtained. The method has already given very important results in studies of yeast (Hereford and Hartwell, '74; Hartwell, '76).

Utilizing miscellaneous clues and cross. checking the conclusions reached with different approaches. It should be clear from the preceding discussion that the available methods for attempting to derive functional sequence maps present a variety of opportunities to draw erroneous conclusions. This fact seems to me to have four general morals. First, it is clearly desirable to include in the analysis as many different mutants and inhibitors as possible; as emphasized repeatedly above, this 
will often allow potentially misleading data to be recognized as such. Second, since the different approaches are subject to somewhat different artifacts, it is clearly desirable to cross-check the conclusions drawn using different approaches. (Note in particular that the reciprocal shift and double mutant methods are affected quite differently by leakiness of the mutants used.) Third, any ancillary evidence that can be adduced to support a proposed sequence is most welcome. IA good example is the use by Hereford and Hartwell (74) of data on the protein synthesis requirements of the $c d c 4$ and $c d c 7$ mutants to support the sequence suggested by the double mutant approach.] Finally, it seems clear that all conclusions about functional sequences, and particularly conclusions in which primary defect events have been translated into gene product synthesis events and gene product function events, should be regarded as tentative until the actual molecular mechanisms of cell cycle progress are known.

\section{LITERATURE CITED}

Basilico, C. 1977 Temperature-sensitive mutations in animal cells. In: Advances in Cancer Research. G. Klein and S. Weinhous, eds. Academic Press, New York, Vol. 24, pp. 223-266.

Coote, J. G., and J. Mandelstam 1973 Use of constructed double mutants for determining the temporal order of ex pression of sporulation genes in Bacillus subtilis. J. Bacteriol., 114: 1254-1263.

Culotti, J., and L. H. Hartwell 1971 Genetic control of the cell division cycle in yeast: seven genes controlling nuclear division. Exptl. Cell Res., 67: 389-401.

Frankel, J., L. M. Jenkins and L. E. DeBault 1976 Causal relations among cell cycle processes in Tetrahymena pyriformis: an analysis employing temperature-sensitive mutants. J. Cell Biol., 71: 242-260.

Hartwell, L. H. 1974 Saccharomyces cerevisiae cell cycle. Bacteriol. Rev., 38: 164-198.

1976 Sequential function of gene products rela- tive to DNA synthesis in the yeast cell cycle. J. Mol. Biol., 104: 803-817.

Hartwell, L. H., J. Culotti, J. R. Pringle and B. J. Reid 1974 Genetic control of the cell division cycle in yeast. Science, 183: 46-51.

Hartwell, L. H., R. K. Mortimer, J. Culotti and M. Culotti 1973 Genetic control of the cell division cycle in yeast: genetic analysis of $c d c$ mutants. Genetics, 74: 267-286.

Hereford, L. M., and L. H. Hartwell 1974 Sequential gene function in the initiation of Saccharomyces cerevisiae DNA synthesis. J. Mol. Biol., 84: 445-461.

Howell, S. H., W. J. Blaschko and C. M. Drew 1975 Inhibitor effects during the cell cycle in Chlamydomonas reinhardtii: determination of transition points in asynehronous cultures. J. Cell Biol., 67: 126-135.

Howell, S. H., and J. A. Naliboff 1973 Conditional mutants in Chlamydomonas reinhardtii blocked in the vegetative cell cycle: an analysis of cell cycle block points. J. Cell Biol., 57: 760-772.

Jarvik, J., and D. Botstein 1973 A genetic method for determining the order of events in a biological pathway. Proc. Natl. Acad. Sci. (U.S.A.), 70: 2046-2050.

1975 Conditional-lethal mutations that sup. press genetic defects in morphogenesis by altering structural proteins. Proc. Natl. Acad. Sci. (U.S.A.), 72. 2783-2742.

Mitchison, J. M. 1971 The Biology of the Cell Cycle. Cambridge University Press, Cambridge.

Morris, N. R. 1976 Mitotic mutants of Aspergillus nidulans. Genet. Res., 26: 237-254.

Nurse, P., P. Thuriaux and K. Nasmyth 1976 Genetic control of the cell division cycle in the fission yeast Schizosaccharomyces pombe. Molec. Gen. Genet., 146: $167-178$.

Orr, E., and R. F. Rosenberger 1976 Determination of the execution points of mutations in the nuclear replication cycle of Aspergillus nidulans. J. Bacteriol., 126: 903-906.

Pringle, J. R. 1975 Induction, selection, and experimental uses of temperature-sensitive and other conditional mutants of yeast. In: Methods in Cell Biology. D. M. Prescott, ed. Academic Press, New York, Vol. 12, pp. 233-272.

Sato, C. 1976 A conditional cell division mutant of Chlamydomonas reinhardii having an increased level of colchicine resistance. Exptl. Cell Res., 101: 251-259.

Siminovitch, L., and L. H. Thompson 1978 The nature of conditionally lethal temperature-sensitive mutations in somatic cells. J. Cell. Physiol., 95: 361-366.

Wells, J. R., and T. W. James 1972 Cell cycle analysis by culture fractionation. Exptl. Cell Res., 75: 465-474. 